\title{
CURRENT CALCULATION OF IRREVERSIBLE ELECTRODE REACTION MECHANISMS IN LINEAR SWEEP VOLTAMMETRY
}

\author{
JÁN MOCÁK, ESTERA RÁBAROVÁ \\ Department of Chemistry, University of SS. Cyril and Methodius, J. Herdu 2, Trnava, \\ SK-917 01, Slovak Republic (jan.mocak@ucm.sk, estera.rabarova@ucm.sk)
}

\begin{abstract}
Application of exponential infinite series gives highly accurate analytical solution contributing to the theory of linear sweep voltammetry for single scan experiments. We have calculated theoretical dimensionless current function (usually denoted as $\pi^{1 / 2} \chi(b t)$ ) at relevant potentials for irreversible charge transfer without a coupled chemical reaction. For this purpose several transformation techniques were used, which convert the derived infinite series into summable sequences. Since infinite series of further electrochemical mechanisms with irreversible electrode reaction have similar features (particularly those comprising preceding and catalytic chemical reaction), the same approach can be successfully applied also for further electrochemical mechanisms.

The respective infinite series are divergent in the most important potential region at and after voltammetric peak therefore their transformation by Epsilon and Levin transform techniques was used. Necessary arbitrary precision arithmetic (APA) was implemented by UBASIC. The results were compared to the customary solution of Nicholson and Shain, who computed the current-potential curves by means of numerical solution of the integral equations but with a much lower precision. Our results were obtained in a broad potential range including the potential regions where the series are divergent. Obtained current functions are precise to 12 valid decimal numbers, which is utilizable for evaluation of the results achieved by various faster but less precise digital simulation techniques.
\end{abstract}

Key words: linear sweep voltammetry, irreversible charge transfer, dimensionless current function, infinite series, arbitrary precision mathematics.

\section{Introduction}

Mathematical description of current - potential curves, which create an essential analytical signal in linear sweep voltammetry, is complex end still not sufficiently treated in electrochemical literature, in spite of its value. The relevant calculations originate from a system of parabolic differential equations describing the concentrations of the reduced $R$ and oxidized $O$ forms of the studied electrochemical system as well as concentrations of further species eventually taking part in the overall electrochemical mechanism. Different mechanisms differ in the detailed composition of initial and boundary conditions. An effective way of calculation submitted Nicholson and Shain (NICHOLSON and SHAIN, 1964) who solved eight electrochemical mechanisms by converting the boundary value problem into integral equations, which may be further solved either by the derived infinite series or directly - using a numerical solution. Of them only the series solution is considered analytical from the mathematical point of view since it provides an arbitrary precise result in the region where the series converge. The main problem with the series solution is that they are generally not convergent in the most desired potential region close to the maximum current (peak), which is basic for quantitative analysis. Therefore very 
many numerical ways of calculation have been described in electrochemical literature, mostly indicated as and belonging to digital simulation (BRITZ, 2005; BRITZ, 2009; RUDOLPH et al., 1994; MOCAK and FELDBERG, 1994).

This work is focussed to the uncomplicated irreversible electrode reaction (mechanism II denoted by NICHOLSON and SHAIN (1964). Severe problems with the series convergence can be overcome (at least in a sufficient extent) by transformation of divergent series into summable sequences.

\section{Theory, problem formulation and tools}

\subsection{Convergence acceleration and resummation}

In applied mathematics, natural and technical sciences, various methods are used for the convergence acceleration of slowly convergent sequences or series and for the summation of divergent series. Their basic idea is to extract hidden information contained in partial sums of a specific slowly convergent or divergent series, and to use that information in order to make a qualified estimate about new (usually higherorder) partial sums which eventually converge to some limit. In many cases, this "qualified estimate" leads to spectacular numerical results which represent a drastic improvement over a term-by-term summation of the original series, even if the series is formally convergent.

For further discussion it is useful to consider a sequence $\left\{\left\{s_{n}\right\}\right\}=\left\{\left\{s_{0}, s_{1}, \ldots\right\}\right\}$ with elements $s_{n}$ or the terms $a_{n}=s_{n}-s_{n-1}$ of an infinite series.

Sequence transformations are important tools for the convergence acceleration of slowly convergent sequences or series and also for the summation of divergent series (BREZINSKI, 2000). The basic idea is to construct from a given sequence $\left\{\left\{s_{n}\right\}\right\}$ a new sequence $\left\{\left\{s_{n}^{\prime}\right\}\right\}=T\left(\left\{\left\{s_{n}\right\}\right\}\right)$ where each $s_{n}^{\prime}$ depends on a finite number of elements $s_{n 1}, \ldots, s_{n m}$. Often, the $s_{n}$ are the partial sums of an infinite series. The aim is to find a transformation $T$ such that $\left\{\left\{s_{n}^{\prime}\right\}\right\}$ converges faster than $s_{n}$ or, after all, it is capable to sum $\left\{\left\{s_{n}\right\}\right\}$. A common approach is to rewrite $s_{n}$ as

$$
s_{n}=s+R_{n}
$$

where $s$ is the limit (or antilimit in the case of divergence) and $R_{n}$ is the remainder. The aim then is to find a new sequence $\left\{\left\{s_{n}^{\prime}\right\}\right\}$ such that

$$
s_{n}^{\prime}=s+R_{n}^{\prime}, \quad R_{n}^{\prime} / R_{n} \rightarrow 0 \text { for } n \rightarrow \infty
$$

Thus, the sequence $\left\{\left\{s_{n}^{\prime}\right\}\right\}$ converges faster to the limit $s$ (or diverges less violently) than $\left\{\left\{s_{n}\right\}\right\}$.

\subsection{Employed ways of series transformation}

A large number of mainly nonlinear sequence transformations for the acceleration of convergence and the summation of divergent series are discussed in review (BREZINSKI, 2000). Some of the sequence transformations are well established 
in mathematical literature; among them Wynn's epsilon algorithm (WYNN, 1956; WYNN, 1966) and Levin's sequence transformations (LEVIN, 1973; SMITH and FORD, 1979; 1982), which were used in this work.

Wynn's epsilon algorithm or Epsilon ( $\left.\varepsilon^{-}\right)$transformation is the nonlinear recursive scheme utilising the partial sums of the original series $\varepsilon_{0}^{(0)}=s_{0}, \varepsilon_{0}^{(1)}=s_{1}, \ldots, \varepsilon_{0}^{(n)}=s_{n}$, and defined by the following equations (WYNN, 1956):

$$
\begin{array}{llrl}
\varepsilon_{-1}^{(n)} & =0, & \varepsilon_{0}^{(n)}=s_{\mathrm{n}}, & n \geq 0 \\
\varepsilon_{k}^{(n)}=\varepsilon_{k-2}^{(n+1)}+1 / \Delta \varepsilon_{k-1}^{(n)}, & & k>0
\end{array}
$$

where the forward difference operator $\Delta$ is applied to superscripts:

$$
\Delta \varepsilon_{k-1}^{(n)}=\varepsilon_{k-1}^{(n+1)}-\varepsilon_{k-1}^{(n)}
$$

Wynn found out that only the elements of the $\varepsilon$ table with even subscripts $(2 n)$ are applicable for the results calculation (they give so-called Shanks transformation) whereas the elements of the $\varepsilon$ table with odd subscripts $(2 n+1)$ are only auxiliary quantities. Each calculation element has a rhombus structure containing four terms and a recursive calculation using a single 1-dimensional array is possible (WYNN, 1965). Epsilon algorithm allows a simple and efficient series transformation and stimulated an enormous amount of research in this field.

Transformations $T\left(\left\{\left\{s_{n}\right\}\right\},\left\{\left\{R_{n}\right\}\right\}\right)$ that depend not only on the sequence elements or partial sums $s_{n}$ but also on an auxiliary sequence containing the estimates of the remainder $R_{n}$ are of Levin-type if they are linear in the $s_{n}$, and nonlinear in the $R_{n}$. Remainder estimates are usually denoted $\omega_{n}$ and provide an easy-to-use possibility to utilize asymptotic information on the problem sequence for the construction of highly efficient sequence transformations. Levin transformations are based on the ratio of two series. The first series (in numerator) is a function of $s_{n}$ values of the original sentence divided by $\omega_{n}$, the second series (in denominator) depends on $1 / \omega_{n}$ (Figure 1). Several variants of Levin transformation differ in the way how $\omega_{n}$ is estimated. In this work two variants were found most useful, particularly Levin $u$ and Levin $d$ transformations. They all can be solved recursively by using only a 3-term recurrence formulas, which are separately expressed for numerator as well as denominator and the final result is achieved as the ratio of the lastly calculated numerator and denominator terms.

$$
L_{k}^{(n)}\left(s_{n}, \omega_{n}\right)=\frac{\sum_{j=0}^{k}(-1)^{j}\left(\begin{array}{l}
k \\
j
\end{array}\right) \frac{(n+1+j)^{k-1}}{(n+1+k)^{k-1}} \frac{s_{n+j}}{\omega_{n+j}}}{\sum_{j=0}^{k}(-1)^{j}\left(\begin{array}{l}
k \\
j
\end{array}\right) \frac{(n+1+j)^{k-1}}{(n+1+k)^{k-1}} \frac{1}{\omega_{n+j}}}
$$

Fig. 1. General calculation scheme of Levin transformations; remainder estimates $\omega_{n}=(n+1) a_{n}$ and $\omega_{n}=a_{n+1}$ for the $u$ and $d$ transformations, respectively. 


\subsection{Solved problems and corresponding series}

Investigated irreversible electrochemical reaction is identical to the mechanisms II defined by Nicholson and Shain (NICHOLSON and SHAIN, 1964) where all employed symbols, common in voltammetry, are defined. The corresponding reaction scheme and the derived series are:

$$
\begin{gathered}
R-z \mathrm{e}^{-} \stackrel{k}{\Rightarrow} O \\
I_{\mathrm{II}}^{*}=\sqrt{\pi} \chi(b t)=\sum_{j=1}^{\infty}(-1)^{j+1} \frac{(\sqrt{\pi})^{j}}{\sqrt{(j-1) !}} \exp \left[\left(\frac{j \beta n_{a} F}{R T}\right)\left(E-E^{0}+\frac{R T}{\beta n_{a} F} \ln \frac{\sqrt{\pi D_{0} b}}{k_{s}}\right)\right]
\end{gathered}
$$

The summation of the series was performed by Epsilon and Levin $u$ transformations (more suitable at larger potential values). Computer program for recursive calculation of this mechanism was written in UBASIC programming language, which allows implementation of arbitrary precision arithmetic (APA) and uses all numbers with very many decimal places (KOYAMA, 2000).

The potential scale utilized by Nicholson and Shain and their successors is defined as:

$$
E^{*}(\mathrm{~N}-\mathrm{S})=\left(E-E^{0}\right) \alpha z_{a}+(R T / F) \ln \left[\left(\pi D_{O} b\right)^{1 / 2} / k_{s}\right]
$$

However, Oldham and coworkers in several important papers concerning irreversible electrode reaction used a simpler potential scale (e.g. in DALRYMPLEALFORD, 1977) where $\pi^{1 / 2}$ was omitted from the argument of logarithm:

$$
E^{*}(\mathrm{Oldh})=\left(E-E^{0}\right) \alpha z_{a}+(R T / F) \ln \left[\left(D_{O} b\right)^{1 / 2} / k_{s}\right]
$$

so that this scale is shifted by $(R T / F) \ln (\pi)^{1 / 2}=14.705546966777 \mathrm{mV}$ or by 0.572364943 when the dimensionless potential scale is used as another alternative, frequently utilized in electrochemistry.

\section{Results and discussion}

\subsection{Current - potential dependence for irreversible electrode reaction using real unit potential scale}

For the sake of space the obtained results are demonstrated in a tabulated form. Table 1 brings the calculated values of the current function $\pi^{1 / 2} \chi(b t)$ designated here also as the dimensionless current $I^{*}$, which was used in our previous papers (MOCAK, 2002; MOCAK and BOND, 2004) and in the pioneer works of Reinmuth. Potential scales of Nicholson-Shain (N-S) as well as Oldham (Oldh) are implemented using real millivolt units.

Our precise values located in the third and seventh column (using N-S scale) can be compared to the values shown in the columns two and six, which were 
calculated numerically 45 years ago by NICHOLSON and SHAIN (1964). One should highly appreciate them (especially considering the computer level that time) since the deviation on the third rounded decimal place is observed only in four cases (emphasized in italics). It is surprising that the results of SIVAKUMAR and BASHA (2005), who investigated the same problem and used four decimal figures for the tabulated current function, were less precise behind the peak potential than the results of Nicholson and Shain and their potential range was even smaller than that of Nicholson and Shain.

Table 1. Calculated dimensionless current for irreversible electrode oxidation using Nicholson-Shain and Oldham potential scales expressed in real millivolt units.

\begin{tabular}{cccccccc}
\hline $\begin{array}{c}E^{*}, \\
\mathrm{mV}\end{array}$ & $\begin{array}{c}\pi^{1 / 2} \chi(b t) \\
(\mathrm{N}-\mathrm{S})\end{array}$ & $\begin{array}{c}I^{*} \\
(\mathrm{~N}-\mathrm{S})\end{array}$ & $\begin{array}{c}I^{*} \\
(\mathrm{Oldh})\end{array}$ & $\begin{array}{c}E^{*}, \\
\mathrm{mV}\end{array}$ & $\begin{array}{c}\pi^{1 / 2} \chi(b t) \\
(\mathrm{N}-\mathrm{S})\end{array}$ & $\begin{array}{c}I^{*} \\
(\mathrm{~N}-\mathrm{S})\end{array}$ & $\begin{array}{c}I^{*} \\
(\mathrm{Oldh})\end{array}$ \\
\hline-300 & & 0.00001505 & 0.00000849 & -10 & 0.462 & 0.46201793 & 0.37464546 \\
-250 & & 0.00010536 & 0.00005944 & -5 & 0.480 & 0.48053005 & 0.40852572 \\
-200 & & 0.00073716 & 0.00041603 & 0 & 0.492 & 0.49178853 & 0.43859990 \\
-160 & 0.003 & 0.00348738 & 0.00197054 & 5 & 0.496 & 0.49574697 & 0.46329925 \\
-150 & & 0.00513823 & 0.00290546 & 10 & 0.493 & 0.49301358 & 0.48139777 \\
-140 & 0.008 & 0.00756454 & 0.00428200 & 15 & 0.485 & 0.48470520 & 0.49222092 \\
-120 & 0.016 & 0.01632951 & 0.00927948 & 20 & 0.472 & 0.47221381 & 0.49576253 \\
-110 & 0.024 & 0.02391140 & 0.01363423 & 25 & 0.457 & 0.45696130 & 0.49266598 \\
-100 & 0.035 & 0.03488516 & 0.01999080 & 30 & 0.441 & 0.44020946 & 0.48407130 \\
-90 & 0.050 & 0.05062198 & 0.02922079 & 35 & 0.423 & 0.42295691 & 0.47137873 \\
-80 & 0.073 & 0.07288591 & 0.04252075 & 40 & 0.406 & 0.40591713 & 0.45600522 \\
-70 & 0.104 & 0.10376342 & 0.06146949 & 45 & & 0.38955001 & 0.43919904 \\
-60 & 0.145 & 0.14535833 & 0.08802158 & 50 & 0.374 & 0.37411700 & 0.42194196 \\
-50 & 0.199 & 0.19905868 & 0.12433319 & 60 & & 0.34644141 & 0.38861355 \\
-45 & & 0.23037469 & 0.14676318 & 70 & 0.323 & 0.32294382 & 0.35892542 \\
-40 & 0.264 & 0.26422510 & 0.17225545 & 80 & & 0.30310390 & 0.33350738 \\
-35 & 0.300 & 0.29992874 & 0.20082435 & 100 & & 0.27192236 & 0.29386329 \\
-30 & 0.337 & 0.33648220 & 0.23230306 & 120 & & 0.24872422 & 0.26514046 \\
-25 & 0.372 & 0.37256762 & 0.26628454 & 150 & & 0.22320466 & 0.23462457 \\
-20 & 0.406 & 0.40661969 & 0.30206924 & 200 & & 0.19446262 & 0.20168254 \\
-15 & 0.437 & 0.43696362 & 0.33863453 & 250 & & 0.17485343 & 0.17997456 \\
\hline
\end{tabular}

\subsection{Current - potential dependence for irreversible electrode reaction using dimensionless potential scale}

In principle the same calculation approach was used for obtaining the dimensionless current $I^{*}$ related to the dimensionless potential $E^{*}$ defined again in two potential scales, those introduced by Nicholson-Shain (N-S) and Oldham (Oldh), respectively. Tabulated results are exhibited in Table 2 . Assuming $T=298.15 \mathrm{~K}$, one dimensionless potential unit corresponds to the value $R T / F=25.692606 \mathrm{mV}$ (MOCAK, 2002).

It is worth mentioning that assuming temperature $T=298.15 \mathrm{~K}$, one dimensionless potential unit corresponds to the value $R T / F=25.692606 \mathrm{mV}$ (MOCAK, 2002). 
Table 2. Calculated dimensionless current for irreversible electrode oxidation using dimensionless Nicholson-Shain and Oldham potential scales.

\begin{tabular}{cccccc}
\hline$E^{*}$ & $I^{*}(\mathrm{Oldh})$ & $I^{*}(\mathrm{~N}-\mathrm{S})$ & $E^{*}$ & $I^{*}(\mathrm{Oldh})$ & $I^{*}(\mathrm{~N}-\mathrm{S})$ \\
\hline-10.0 & 0.00004540 & 0.00008046 & 0.5 & 0.48846716 & 0.48888145 \\
-9.0 & 0.00012339 & 0.00021869 & 1.0 & 0.49177335 & 0.45470458 \\
-8.0 & 0.00033535 & 0.00059424 & 1.5 & 0.46069675 & 0.37002530 \\
-7.0 & 0.00091105 & 0.00161366 & 2.0 & 0.41718128 & 0.30856070 \\
-6.0 & 0.00247262 & 0.00437423 & 3.0 & 0.34050384 & 0.26830872 \\
-5.0 & 0.00669276 & 0.01180127 & 4.0 & 0.28933513 & 0.24060300 \\
-4.0 & 0.01798448 & 0.03143348 & 5.0 & 0.25529883 & 0.22028455 \\
-3.0 & 0.04739313 & 0.08092026 & 6.0 & 0.23121011 & 0.20457946 \\
-2.0 & 0.11864417 & 0.19088973 & 7.0 & 0.21311328 & 0.19194529 \\
-1.0 & 0.26145051 & 0.36765286 & 8.0 & 0.19886406 & 0.18147266 \\
-0.5 & 0.35430163 & 0.44848739 & 9.0 & 0.18724114 & 0.17259233 \\
0.0 & 0.43859990 & 0.49178853 & 10.0 & 0.17750549 & 0.17750549 \\
\hline
\end{tabular}

\section{Conclusions}

The infinite series solution represents at present time the only alternative of analytical solution (from mathematical standpoint) of the corresponding set of differential equations pertinent to irreversible charge transfer (6) in stationary electrode voltammetry and facilitates very precise calculation of voltammetric current even in the potential region far after the peak. Even though the corresponding infinite series (7) behaves very badly at large positive potentials, Levin transformations and the use of arbitrary precision arithmetic prevents catastrophic loss of precision in calculation and enables very accurate calculation even at $250 \mathrm{mV}$ (using N-S scale). In the indicated potential region all calculated dimensionless current values were precise at minimum to 12 decimal places even though the tables exhibited above contain only the numbers with 8 decimal places to be sufficiently lucid. For practical comparison with the calculations obtained in other mathematical ways even a smaller number of decimal places is needed. In any case, the resulting current - potential data obtained in this work may serve as the standard values utilizable for precision assessment of other, perhaps faster computational tools like digital simulation.

Acknowledgement: The support of this work by Slovak Grant Agencies by means of grants VEGA 1/1005/09 and 1/0066/09, and VVCE-0004-07 is highly acknowledged.

\section{References}

BREZINSKI, C.: Convergence acceleration during 20th century. J. Comput. Appl. Math., 122, 2000, 1-21.

BRITZ, D.: Digital simulation in electrochemistry. Lect. Notes Phys., 666, 3rd Edition, Springer-Verlag, Berlin, 2005, 338 pp. 
BRITZ, D.: Digital simulation in electrochemistry, 3rd Ed. Electronic version: http://www.scribd.com/doc/17687737/Digital-Simulation-in-Electrochemistry-3rdEd-Dieter-Britz, 2009.

KOYAMA, Y.: UBasic. Kanazawa Univ. Japan, ftp://rkmath/rikkyolac.jp/pub/ubibm; UBasic ver. 8.8f, download: http://www.rkmath.rikkyo.ac.jp/ kida/ubasic.htm, 2000.

LEVIN, D.: Development of non-linear transformations for improving convergence of sequences. Int. J. Comput. Math., B 3, 1973, 371-388.

MOCAK, J., FELDBERG, S.W.: The Richtmyer modification of the fully implicit finite-difference algorithm for simulations of electrochemical problems. J. Electroanal. Chem., 378, 1994, 31-37.

MOCAK, J.: Voltammetric current-potential calculations using infinite series solution. Electrochem. Comm., 4, 2002, 803-807.

MOCAK, J., BOND, A.M.: Use of MATHEMATICA software for theoretical analysis of linear sweep voltammograms. J. Electroanal. Chem., 561, 2004, 191-202.

NICHOLSON, R.S., SHAIN, I.: Single scan and cyclic methods applied to reversible, irreversible, and kinetic systems. Anal. Chem., 36, 1964, 706-723.

DALRYMPLE-ALFORD, P., GOTO, M., OLDHAM, K.B.: Shapes of derivative neopolarogram. J. Electroanal. Chem., 55, 1977, 1-15.

RUDOLPH, M., REDDY, D.P., FELDBERG, S.W.: A simulator for cyclic voltammetric responses. Anal. Chem., 66, 1994, A589-A600.

SIVAKUMAR, S., BASHA, C.A.: Evaluation of the current function in linear sweep voltammetry by Pade approximation and epsilon convergence. Russian J. Electrochem., 41, 2005, 421-438.

SMITH, D.A., FORD, W.F.: Acceleration of linear and logarithmic convergence. SIAM J. Numer. Anal., 16, 1979, 223-240.

SMITH, D.A., FORD, W.F.: Numerical comparisons of nonlinear convergence accelerators. Math. Comput., 38, 1982, 481-499.

WYNN, P.: On a device for computing the $\operatorname{em}(S n)$ transformation. Math. Tables Aids Comput., 10, 1956, 91-96.

WYNN, P.: A note on programming repeated applications of the $\varepsilon$-algorithm. Rev. Franc. Trait. Inf. - Chiffres, 8, 1965, 23-62.

WYNN, P.: On the convergence and the stability of the epsilon algorithm. SIAM J. Numer. Anal., 3, 1966, 91-122. 